\title{
Involvement of Nitric Oxide System in Experimental Muscle Crush Injury
}

\author{
Irit Rubinstein, Zaid Abassi, Raymond Coleman, Felix Milman, Joseph Winaver, and Ori S. Better \\ Department of Physiology and Biophysics and R. Chutick Crush Research Center, Bruce Rappaport Faculty of Medicine, Technion-Israel \\ Institute of Technology, 31096 Haifa, Israel
}

\begin{abstract}
Muscle crush injury is often complicated by hemodynamic shock, electrolyte disorders, and myoglobinuric renal failure. In this study, we examined the involvement of the nitric oxide (NO) system in the development of muscle damage in an experimental model of crush injury induced by exertion of standardized mechanical pressure on tibialis muscle of rat. The intact limb served as a control. Four days after injury, the crushed muscle was characterized by extreme capillary vasodilatation as demonstrated by histological morphometric analysis. These changes were accompanied by muscle hyperperfusion as evaluated by measurements of femoral blood flow (ultrasonic flowmetry) and capillary blood flow (laser-doppler flowmetry). Treatment with $\mathrm{N \omega}$ nitro-L-arginine methyl ester, a NO synthase (NOS) inhibitor, largely decreased the hyperperfusion. Furthermore, the expression of the different NOS isoforms, assessed by reverse transcription-PCR and immunoreactive levels, determined by Western blot, revealed a remarkable induction of the inducible NOS in the crushed limb. Similarly, endothelial NOS mRNA increased gradually after the induction of muscle damage. In contrast, the major muscular NOS, i.e., neuronal isoform remained unchanged. In line with the alterations in the mRNA levels, Western blot analysis revealed parallel changes in the immunoreactive levels of the various NOS. These findings indicate that muscle crush is associated with activation of the NO system mainly due to enhancement of iNOS. This may contribute to NO-dependent extreme vasodilatation in the injured muscle and aggravate the hypovolemic shock after crush injury. (J. Clin. Invest. 1998. 101:1325-1333.) Key words: crush syndrome • capillary $\bullet$ nitric oxide synthase $\bullet$ RT-PCR $\bullet$ rat
\end{abstract}

Portions of this work were presented at the 29th annual meeting of the American Society of Nephrology, New Orleans, LA, November 1996, and have been published in abstract form (J. Am. Soc. Nephrol. 1996. 7:1833).

Address correspondence to Irit Rubinstein, D.Sc., Department of Physiology and Biophysics, The Bruce Rappaport Faculty of Medicine, Technion-Israel Institute of Technology, P.O. Box 9649, 31096 Haifa, Israel. Phone: 972-4-829-5263; FAX: 972-4-852-0751; E-mail: iritru@tx.technion.ac.il

Received for publication 5 June 1997 and accepted in revised form 12 January 1998.

J. Clin. Invest.

(C) The American Society for Clinical Investigation, Inc. 0021-9738/98/03/1325/09 \$2.00

Volume 101, Number 6, March 1998, 1325-1333

http://www.jci.org

\section{Introduction}

Extensive muscle trauma classically occurs in casualties of mass disasters. Muscle crush injury may also occur after penetration into muscle by high-velocity projectiles, blunt external trauma, or even by gravity during prolonged immobilization in comatose patients after head trauma or alcoholic or drug overdose (1). Muscle crush injury is a limb- and life-threatening condition. When extensive, it may culminate in the crush syndrome, which consists of profound hypovolemic shock, aggravated by gross electrolyte disorders (hyperkalemia, hypocalcemia, and acidosis), ultimately leading to myoglobinuric acute renal failure (2-4).

The mechanism of the myopathy in crush injury is not clear. Traditionally, crush myopathy has been attributed to ischemia resulting from the decrease in blood supply to the injured muscle. This approach, however, cannot explain the appearance of the symptoms after a relatively short period of mechanical pressure. Thus, total ischemia of 3-4 h, as is often practiced in orthopedic surgery, is well tolerated by muscles. Another suggestion is that the tension exerted on the sarcolemma during the crushing of the muscle may stimulate nonselective stretch-activated channels. Opening of these channels would flood the cytosol of the myocyte with cations like $\mathrm{Na}^{+}$ and $\mathrm{Ca}^{2+}$ down their electrochemical gradient, followed by water, and cause cell swelling $(4,5)$. Increased cytosolic calcium activates autolytic processes that may damage the myocyte. The sodium-potassium pumps are maximally activated to restore cell volume, and rapidly deplete cellular ATP stores, and thus undermine myocytic homeostatic capacity. Under these conditions the muscular tissue becomes susceptible to hypoxia despite an initially intact circulation.

Recently, a novel regulator of skeletal muscle function has been described. This regulator is the nitric oxide $(\mathrm{NO})^{1}$ system. It has been demonstrated that NO regulates contractile function of skeletal muscle $(6,7)$, blood vessel tone, and blood flow $(8,9)$. Among the three known isoforms of NO synthase (NOS), rat skeletal muscle contains two constitutive types: neural NOS (nNOS) and, to a lesser extent, endothelial NOS (eNOS) $(6,7,10)$. nNOS is located mostly in the sarcolemma (6) and has been detected predominantly in fast (type II) muscle fibers. eNOS is located in the vascular endothelium of all mammals, but its immunoreactivity has also been detected in the cytoplasm of mitochondria-rich fibers and within the mitochondria of rat skeletal muscle $(7,11)$. Its expression is not re-

1. Abbreviations used in this paper: $\mathrm{CBF}$, capillary blood flow; FBF, femoral blood flow; G3PDH, glyceraldehyde-3-phosphate dehydrogenase; L-NAME, $N \omega$-nitro-L-arginine methyl ester; D-NAME, $N \omega$ nitro-D-arginine methyl ester; MAP, mean arterial pressure; NO, nitric oxide; NOS, NO synthase; eNOS, endothelial NOS; iNOS, inducible NOS; nNOS, neural NOS; PU, perfusion units; RIO, reactive oxygen intermediates; RT-PCR, reverse transcription-PCR. 
lated to fiber type, but it has a striking correlation to mitochondrial content $(7,11)$. Recently, it was reported that after endotoxin administration, mouse skeletal muscle shows the ability to induce the macrophage (inducible) form of NOS (iNOS) mRNA and protein and possesses iNOS activity as well (12).

NOS activity in rat skeletal muscles is correlated with type II muscle fiber density (6). There is an inverse correlation between NOS activity and force development in muscles: synthesis of NO in active muscle opposes contractile force $(6,13)$. $\mathrm{NO}$ is also known as a regulator of blood vessel diameter and blood flow $(8,9)$. Therefore, increased NO production in skeletal muscle can modulate the vascular tone and blood flow in the muscle, as well as its contractile properties. The NOrelated alterations in blood flow might have a further influence on muscle function or even on the systemic circulation and volume control. For example, extreme vasodilatation may contribute to the hemodynamic-hypovolemic shock that is the hallmark of the crush syndrome in humans. Increased NO formation (by induction of iNOS) may also cause devastating damage to the muscular tissue (14) and thus aggravate and possibly propagate rhabdomyolysis. In light of this information on the role of NO, we speculated that NO might be involved in the pathogenesis of muscle crush injury.

This study was designed to test this hypothesis and to evaluate the mechanism(s) of muscle damage in a rat model of muscle crush injury and its complications-in particular, to examine a possible involvement of the NO system in the mechanism of the myopathy.

\section{Methods}

Studies were conducted on Sprague-Dawley rats weighing 220-270 g, maintained on standard rat diet and tap water ad lib. Special care was taken to prevent pain during the induction of crush injury and the other treatments, according to the Technion Committee for the Care and Use of Laboratory Animals.

\section{The experimental model}

Muscle damage was performed in an experimental model of muscle crush injury, developed in our laboratory. Rats were anesthetized by Nembutal (pentobarbital sodium; Ceva, Paris, France), $30 \mathrm{mg} / \mathrm{kg}$ intraperitoneally. The hind limb of the rat was subjected to the same standardized external mechanical pressure, using an apparatus designed for this purpose. The apparatus contains a piston driven by calibrated air pressure with a $4.0-\mathrm{cm}^{2}$ Perspex disc on its end, connected to a metal stand. According to the qualities of this piston, a pressure of one atmosphere is applied on the limb $1.7 \mathrm{~kg} / \mathrm{cm}^{2}$. For induction of crush injury, a pressure of 2.5 absolute atmospheres $\left(4.25 \mathrm{~kg} / \mathrm{cm}^{2}\right)$ was applied for $2 \mathrm{~h}$ on the left lower limb of the rat.

Rats were divided into three groups: $(a)$ rats subjected to experimental muscle crush injury without any further treatment; $(b)$ rats with experimental muscle crush injury and NOS blockade by $N \omega$ nitro-L-arginine methyl ester (L-NAME) per os (100 mg/liter in drinking water for $4 \mathrm{~d}$ after the crush); and (c) control group: normal rats. In the first two groups, the counterpart uninjured limb served as internal control.

To exclude the possibility that L-NAME is acting nonspecifically as an antioxidant (15) rather than via NOS inhibition, we used the stereoisomer, $N \omega$-nitro-D-arginine methyl ester (D-NAME), which does not affect the NOS isoforms and should be inactive in this system. We checked it on a small group and the protocol of treatment was identical to that of L-NAME (group 2).

\section{Histology and capillary morphometry}

Rats were killed immediately following crush or $4 \mathrm{~d}$ later, and the abdominal aorta was cannulated and injected with Microfil silicone radiopaque rubber compound (Flow Tek, Inc., Boulder, CO) at a constant controlled rate and under pressure ranging between 100-120 $\mathrm{mmHg}$. This pressure does not affect the vessels' diameter (16) and was verified using a manometer. After polymerization $(24 \mathrm{~h})$, the tibialis anterior muscles were removed and fixed in $10 \%$ neutral buffered formaldehyde, $\mathrm{pH} 7.4$, and routinely embedded in wax for histological examination. Sections $(6 \mu \mathrm{m})$ cut on a microtome (rotary microtome 50-A standard model; Lipshaw Manufacturing Co., Detroit, MI) were stained with standard hematoxylin and eosin. In parallel, small blocks of the muscle tissue were fixed in 3\% glutaraldehyde in $0.1 \mathrm{M}$ sodium cacodylate buffer, followed by $1 \%$ osmium tetroxide and embedding in epoxy resin (Eponate12 ${ }^{\mathrm{TM}}$; Pelco International, Redding, CA). Ultramicrotome sections (1 $\mu \mathrm{m})$ were stained in $0.1 \%$ toluidine blue in $1 \%$ sodium tetraborate.

Image analysis was undertaken on the histological transverse sections to determine the cross-sectional area of blood capillaries. Quantitative determinations were performed using an Olympus Cue-2 image analysis system with appropriate morphometry software (Olympus Corp., Lake Success, NY). The system consists of a Zeiss Universal R photomicroscope ( $\times 16$ objective), fitted with a Panasonic WV-CD50 camera with the video image seen on a Sony 14 -inch color monitor, and an IBM-compatible PC computer. Measurements were made in standardized windows with an area of $78,840 \mu \mathrm{m}^{2}$.

For capillary morphometry, 4-5 slides were randomly selected from each muscle. Ten capillaries were measured in each slide: 4-5 capillaries from around a muscle fiber, and the rest chosen randomly.

\section{Blood flow measurements}

For hemodynamic measurements, rats were anesthetized $4 \mathrm{~d}$ after the crush using Inactin (thiobutabarbital sodium salt; R.B.I., Natick, MA; $100 \mathrm{mg} / \mathrm{kg}$ intraperitoneally), and placed on a heated surgical table to maintain body temperature at $37^{\circ} \mathrm{C}$. After tracheostomy, polyethylene catheters $\left(\mathrm{PE}_{50}\right)$ were inserted into carotid artery and jugular vein, for measurements of mean arterial pressure (MAP) and infusion of $0.9 \%$ saline. MAP was continuously monitored using a pressure transducer (model 156PC05GWL; Microswitch, Freepoint, IL). Saline was infused throughout the experiment, at a rate of $1.5 \%$ of body wt/h.

The blood flow, either femoral or capillary, was measured simultaneously in left and right hind limbs of each group.

Femoral blood flow $(F B F)$. The femoral arteries of both limbs were exposed by surgical dissection. FBF was measured by a dualchannel ultrasonic flowmeter (model T-206; Transonic Systems, Inc., Ithaca, NY), using perivascular flow probes (type 1R for vessel o.d. $0.5-1 \mathrm{~mm}$ ) loosely encircled around the arteries, to measure the volume flow directly $(\mathrm{ml} / \mathrm{min})$. Recordings were obtained continuously for $30 \mathrm{~min}$ after an equilibration period of 15-30 min.

Data of FBF and MAP were recorded by a computerized data acquisition system using the Labtech-acquired software.

Capillary blood flow $(C B F)$. The tibialis anterior muscles of both limbs were exposed by fine incision of the skin and removal of the fascia. Measurements were performed by multichannel laserDoppler flowmeter (PeriFlux 4001 Master; Perimed AB, Jarfalla, Sweden) at a wavelength of $780 \mathrm{~nm}$, using two probes (PF 415:42) with fiber separation of $0.5 \mathrm{~mm}$. For measurement of capillary perfusion, the probes were placed perpendicular to the surfaces of the muscles at 12-15 different points all over the muscle. The CBF was calculated, using Perisoft software (version 5.09), by multiplying the concentration of moving blood cells (concentration units [CU]) by mean velocity of the cells (velocity units [VU]) and expressed as perfusion units $(\mathrm{PU}): \mathrm{PU}=(\mathrm{CU} \times \mathrm{VU} / 100)$.

\section{Determination of NOS isoforms}

$m R N A$ expression by reverse transcription-PCR (RT-PCR) method. Tissues (tibialis anterior muscles) from both hind limbs (normal and 


\begin{tabular}{|c|c|c|c|}
\hline & & Primer sequences & Size (bp) \\
\hline \multirow[t]{4}{*}{$\mathrm{nNOS}$} & Upstream: & 5'-AGGTTCCTGCAGGAGTCATT - 3' & 709 \\
\hline & Bases: & $(298-317)$ & \\
\hline & Downstream: & 5' - TGTTGGAAGCTTTGCTCCCACTGTTGAGGATG - 3' & \\
\hline & Bases: & $(975-1006)$ & \\
\hline \multirow[t]{4}{*}{ eNOS } & Upstream: & 5' - CCGGAATTCGAATACCAGCCTGATCCATGGAA - 3' & 614 \\
\hline & Bases: & $(2456-2487)$ & \\
\hline & Downstream: & 5' - GCCGGATCCTCCAGGAGGGTGTCCACCGCATG - 3' & \\
\hline & Bases: & $(3038-3069)$ & \\
\hline \multirow[t]{4}{*}{ iNOS } & Upstream: & $5^{\prime}$ - GCATGGACCAGTATAAGGCAAGCA - 3' & 222 \\
\hline & Bases: & $(1693-1716)$ & \\
\hline & Downstream: & 5' - GCTTCTGGTCGATGTCATGAGCAA - 3' & \\
\hline & Bases: & $(1892-1915)$ & \\
\hline \multirow[t]{4}{*}{ G3PDH } & Upstream: & 5' - TCTACTGGCGTCTTCACCACC - 3' & 670 \\
\hline & Bases: & $(286-306)$ & \\
\hline & Downstream: & 5' - TGTAGCCATATTCATTGTCATACC - 3' & \\
\hline & Bases: & $(932-955)$ & \\
\hline
\end{tabular}

crushed) were harvested at $6,24,48$, and $72 \mathrm{~h}$ after the induction of crush injury, and immediately placed in liquid nitrogen. RNA was extracted from the tissues as described by Chomczyski and Sacchi (17), after homogenization in a commercial solution (RNAzol B; Tel-Test Inc., Friendswood, TX), and was quantified by absorbance spectrophometry at $260 \mathrm{~nm}$. mRNA levels were assessed by RT followed by PCR.

cDNAs for nNOS, eNOS, and iNOS, were synthesized from $2 \mu \mathrm{g}$ of total RNA using specific primers (Table I).

Avian myeloblastosis virus reverse transcriptase (AMV-RT, $16 \mathrm{U}$ per reaction; Promega Corp., Madison, WI) was used for RT, along with the reaction mixture recommended by the enzyme manufacturer, in a volume of $20 \mu \mathrm{l}$, using $1.25 \mu \mathrm{M}$ downstream primer. PCR was applied with $2 \mu \mathrm{l}$ of the resulting cDNA using $1.25 \mu \mathrm{M}$ of both the upstream and the downstream primers. Each PCR reaction mixture contained $1.5 \mathrm{U}$ of the enzyme Taq polymerase (Takara Ex Taq ${ }^{\mathrm{TM}}$; TakaRa Biomedical Europe S.A., Gennevilliers, France) and $60 \mu \mathrm{M}$ dNTP. Primers were chosen to span introns that would distinguish by size PCR products derived from cDNA, from those derived from genomic DNA contaminants. In a preliminary study, we found that 30-33 of PCR cycles were necessary for the three NOS isoforms to obtain a visible product in an agarose gel, which was in proportion to the amount of cDNA used. After an initial denaturation step at $94^{\circ} \mathrm{C}$ for $2 \mathrm{~min}$, cycles of annealing at $56^{\circ} \mathrm{C}$ for $45 \mathrm{~s}$, elongation at $72^{\circ} \mathrm{C}$ for $90 \mathrm{~s}$, and denaturation at $94^{\circ} \mathrm{C}$ for $45 \mathrm{~s}$ were performed with $10 \%$ of the cDNA $(2 \mu \mathrm{l})$, as described above. The RT-PCR product of the gene encoding the glycolytic enzyme glyceraldehyde-3-phosphate dehydrogenase (G3PDH) served as a quantity control (18), and it required 25 cycles to obtain a visible product.

$5 \mu \mathrm{l}$ of PCR products were electrophoresed on agarose TAE gel. The resulting gel was stained with ethidium bromide $(0.5 \mu \mathrm{g} / \mathrm{ml})$ for 15 min until clear bands were visible in ultraviolet light.

Relative muscle tissue levels of mRNA encoding the different NOS isoforms were quantified by densitometry, using NIH image software and normalized to G3PDH mRNA.

Analysis of immunoreactive levels of NOS isoforms by Western blot technique. Tissues were removed as described for the RT-PCR procedure and resuspended in $2.5 \mathrm{ml}$ of $10 \mathrm{mM}$ sodium phosphate buffer, $\mathrm{pH} 7.4$, containing $1 \mathrm{mM} \mathrm{MgCl}, 30 \mathrm{mM} \mathrm{NaCl}, 0.02 \%$ sodium azide, $20 \mathrm{mg} /$ liter bestatin, and $20 \mathrm{mg} /$ liter leupeptin. The tissue was then homogenized with a $3 \times 30$ inch polytron homogenizer $(\times 620$ CAT, D-7813 Staufen, Germany). Kaleidoscope prestained standards molecular markers (Bio-Rad Laboratories, Hercules, CA) were used for determination of the molecular weight of the immunoreactive products. $10 \mu \mathrm{l}$ of the tissue homogenate $(120 \mu \mathrm{g}$ protein) were treated with $20 \mu \mathrm{l}$ sample buffer (10\% SDS, 50\% glycerol, $1 \mathrm{M}$ Tris, $0.1 \%$ bromphenol blue, and $1 \mathrm{M}$ DTT, $\mathrm{pH} 6.8$ ) and placed in a boiling water bath for $5 \mathrm{~min}$. Samples were then electrophoresed on polyacrylamide, Tris-glycine gels 4\%-20\% (Bio-Rad Laboratories), and transferred electrophoretically to a nitrocellulose membrane $(150 \mathrm{~V}$ for 45-60 $\mathrm{min})$. The nitrocellulose membrane was incubated with NOS mAb (Transduction Laboratories, Inc., Lexington, KY): nNOS, 155 kD (IgG2a); eNOS, 140 kD (IgG1); and iNOS, 130 kD (IgG2a). Brain, endothelial, and macrophage lysates (Transduction Laboratories, Inc.) were used as positive controls for nNOS, eNOS, and iNOS, respectively. Bands were visualized by successive incubation with goat anti-mouse IgG-alkaline phosphatase conjugate, and alkaline phosphate buffer with color reagents (Bio-Rad Laboratories).

\section{Statistical analysis}

To compare values of control and experimental groups we used ANOVA, followed by Dunnett test (Instat2 software, version 2.04, GraphPad). $P<0.05$ was considered statistically significant. Data are presented as means \pm SEM.

\section{Results}

Muscle histopathology and capillary morphometry. $4 \mathrm{~d}$ after standardized crush, the tibialis anterior muscle was grossly edematous. This were reflected in many swollen myofibers (Fig. 1). The myofibers demonstrated marked myopathy consisting of disruption of sarcomeres and patchy distortion or disappearance of striation. The myocytes frequently showed larger, clear central vacuoles. Large lipid inclusions were common, particularly near the sarcolemma. The nuclei of myofibers typically had very large and pronounced nucleoli, and there was a tendency for the nuclei to become centralized in the myofibers. In the more severely affected regions of damaged and necrotic myofibers, macrophages were present in the vicinity of the myofibers and commonly contained numerous lipid inclusions, presumably from breakdown products of degenerating fibers. 


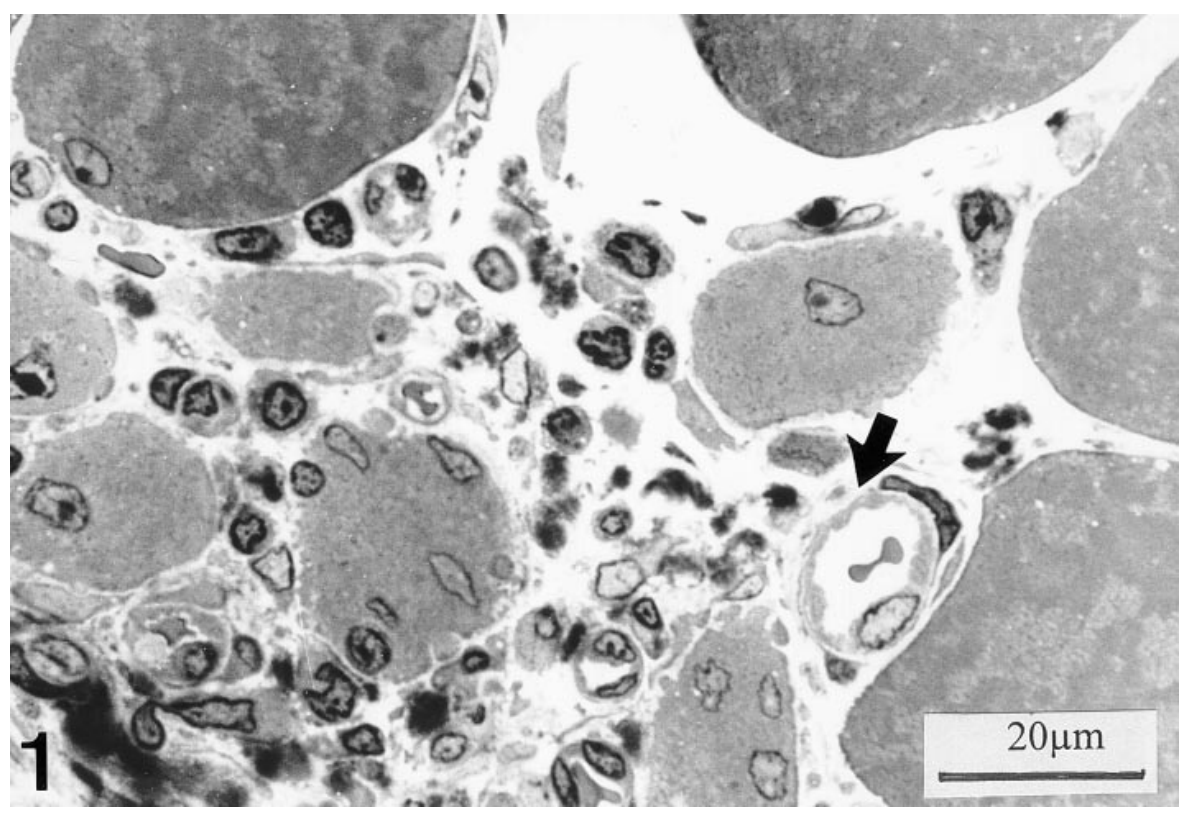

Figure 1. Details of tibialis anterior muscle $4 \mathrm{~d}$ after crush trauma, showing infiltration of macrophages and PMN and activation of satellite cells. Capillaries may be dilated (arrow). In regenerating myofibers, the nuclei have become centralized (1- $\mu \mathrm{m}$ Epon section, stained with alkaline toluidine blue).
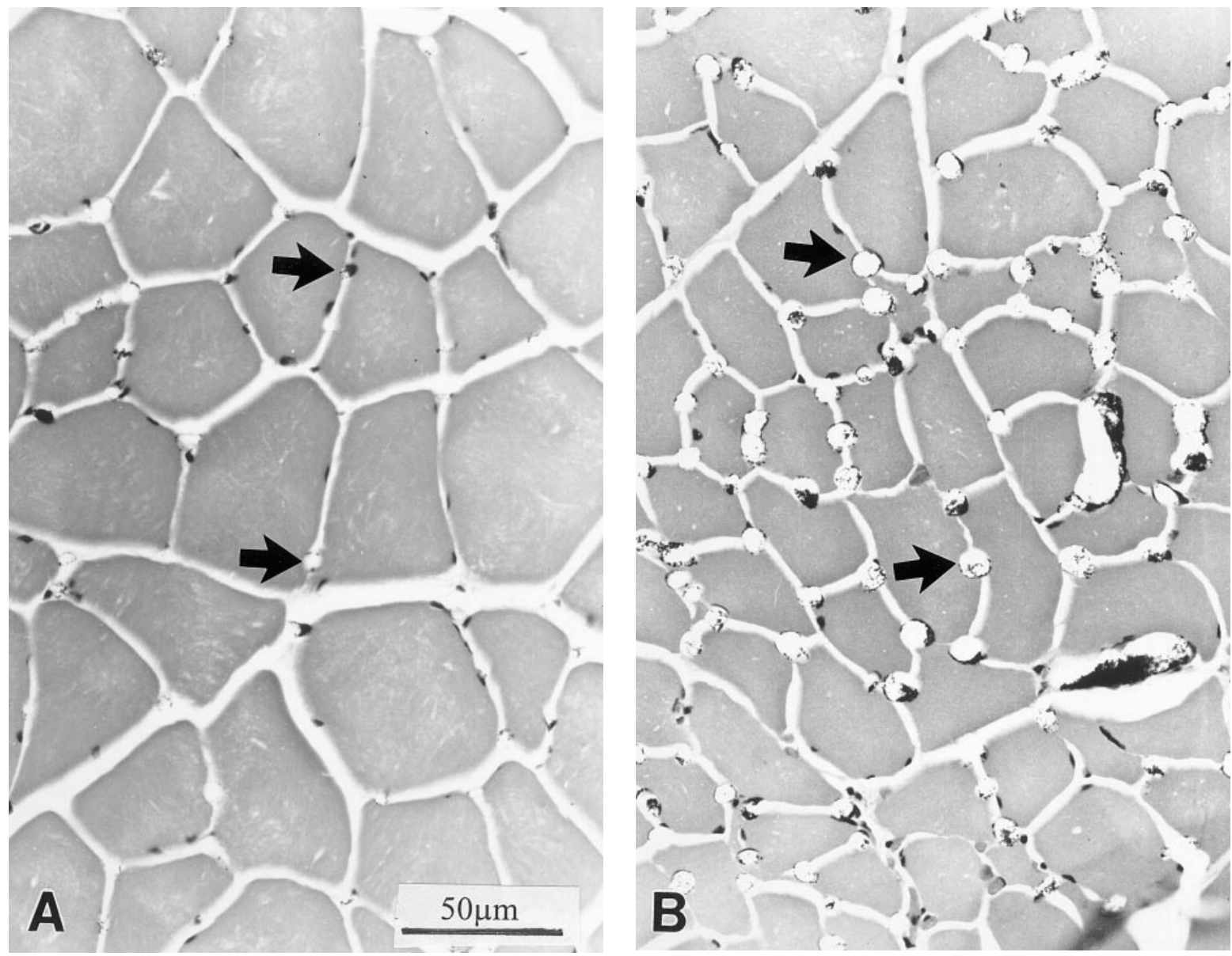

Figure 2. Transverse section of tibialis anterior muscles to show response of capillaries (arrows) to crush trauma. (A) Control (contralateral leg); $(B)$ crush-damaged leg ( $4 \mathrm{~d}$ after trauma), showing vasodilatation of capillaries (6- $\mu \mathrm{m}$ wax sections, stained with hematoxylin and eosin). The vascular system was injected with radiopaque material before embedding. 
The connective tissue of the crush area was marked by massive infiltration and accumulation of macrophages and PMN (Fig. 1). The capillaries associated with the myofibers remained intact, although most of them were clearly dilated in many cases. There were increased numbers of satellite cells in the connective tissue and adjacent to myofibers. These satellite cells could be identified by their regular nuclei and prominent nucleoli. There were also indications of activation of perivascular cells (pericytes) associated with capillaries.

$4 \mathrm{~d}$ after the crush there were indications of the early stages of myofiber regeneration, and myotubes with strings of central nuclei were frequently seen.

Fig. 2 shows representative photographs of transverse sections of tibialis anterior muscle, in a normal $(A)$ and crushed $(B)$ limb $72 \mathrm{~h}$ after crushing. The capillaries in normal strained muscle surrounded each myofibril. Most of them ran between and parallel to the myofibrils in the endomysium. In cross section, individual capillaries appeared as delicate rings, either empty or containing a red blood cell. Crescentic nucleus of the endothelial cells might appear attached to the blood cells. An average of four vessels was found to supply each fiber, in a square or hexagonal arrangement, which in some way constitutes the optimal number of vessels for diffusion of oxygen into a cylindrical muscle fiber $(16,19)$.

As shown in Fig. 2, the major difference before and $72 \mathrm{~h}$ after crush injury related to the diameter of the capillaries. At this time point, the tibialis anterior muscle in the crushed limb was characterized by extreme vasodilatation. No changes were observed in the counterpart, uninjured limb (data not shown), therefore the vasodilatation was due to the crush injury and not a result of the microfil injection.

The measurements of capillary mean cross-sectional area are shown in Fig. 3. In the crushed untreated group, the mean cross-sectional area was approximately threefold that observed in normal $\left(252.44 \pm 6.07\right.$ versus $88.11 \pm 3.98 \mu \mathrm{m}^{2}, P<$ 0.001). Treatment with L-NAME completely prevented the extreme vasodilatation caused by crush, and the mean crosssectional area decreased significantly below normal (66.15 $\left.2.11 \mu \mathrm{m}^{2}, P<0.001\right)$.

Blood flow measurements. Fig. 4 demonstrates the FBF in the three groups. In the crushed-untreated group, FBF was

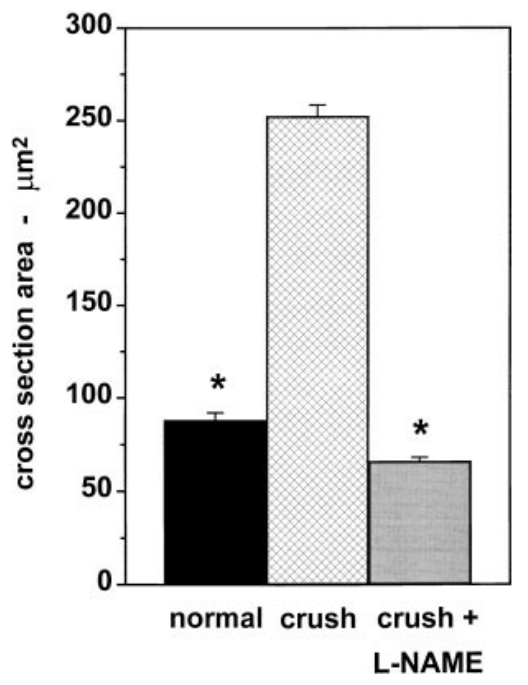

Figure 3. Capillary mean cross-sectional area $\left(\mu \mathrm{m}^{2}\right)$ in tibialis anterior muscle of $(a)$ normal rats; (b) $4 \mathrm{~d}$ after crush injury without treatment; and (c) rats after crush injury and L-NAME treatment $* P<0.001$ compared with crush injury without treatment. Data are based on measurement of 200-240 capillaries in each group.

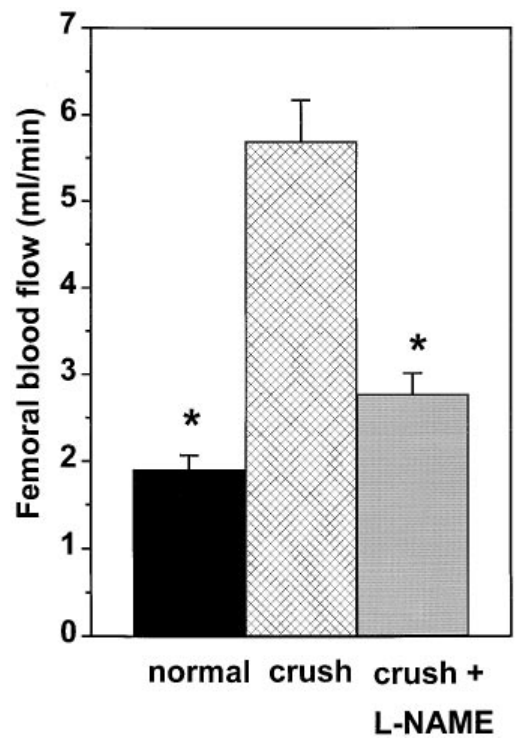

Figure 4. $\mathrm{FBF}(\mathrm{ml} / \mathrm{min})$ in normal rats $(n=17)$, in rats after crush injury without treatment $(n=19)$, and in rats after crush injury and L-NAME treatment $(n=12) . * P<0.001$ compared with crush injury without treatment.

about threefold greater than in the normal group (5.7 \pm 0.47 versus $1.91 \pm 0.16 \mathrm{ml} / \mathrm{min}, P<0.001)$. This increased blood flow in the injured limb was commensurate with the extreme vasodilatation found in capillary morphometry. L-NAME decreased this hyperperfusion significantly $(2.78 \pm 0.24 \mathrm{ml} / \mathrm{min}$, $P<0.001)$. A similar decrease in perfusion after treatment with L-NAME was observed in counterpart untreated limbs (see also Table II).

Fig. 5 shows the CBF in the same groups. The flow in the crushed untreated group was about fourfold higher compared with the control group (278.55 \pm 14.9 versus $70.32 \pm 4.7 \mathrm{PU}, P<$ 0.001). This hyperperfusion decreased significantly $(P<0.001)$ after treatment with L-NAME (152.74 \pm 5.2 PU); however, it remained significantly higher than normal $(P<0.01)$.

After treatment with D-NAME, CBF in the intact and crushed limbs were $66.99 \pm 2.16$ and $243.35 \pm 12.46$ (mean \pm SEM) respectively $(n=4)$. There was no statistical significance of the mean compared with crushed untreated rats, but $P<0.001$ compared with crushed plus L-NAME rats.

Table II summarizes the FBF and CBF in the counterpart uninjured limb. No changes in blood flow were found in the crushed untreated group compared with control. Furthermore, FBF and CBF in the uninjured limb in the crushed untreated group were the same as in normal rats (data not shown). In contrast to the crushed limb, L-NAME decreased FBF in the counterpart uninjured limb significantly, to lower values than

Table II. Blood Flow in the Counterpart Untreated Limb

\begin{tabular}{lccc}
\hline & Normal & Crush-untreated & Crush + L-NAME \\
\hline FBF (ml/min) & & & \\
No. of rats & 17 & 19 & 12 \\
Mean \pm SEM & $2.06 \pm 0.17$ & $2.18 \pm 0.16$ & $1.41 \pm 0.15^{*}$ \\
CBF (PU) & & & 10 \\
No. of rats & 10 & 10 & $63.56 \pm 2.7$ \\
Mean \pm SEM & $69.4 \pm 3.7$ & $79.35 \pm 7.2$ & \\
\hline
\end{tabular}

$* P<0.05$ versus normal. 


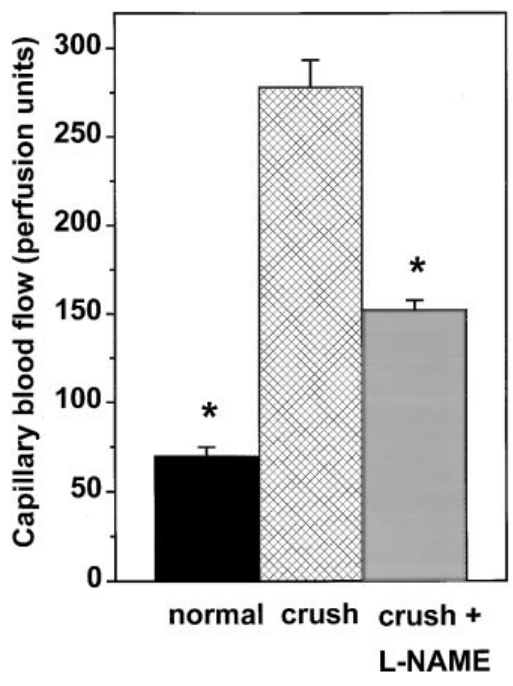

Figure 5. CBF (PU) in normal rats, in rats after crush injury without treatment, and in rats after crush injury and L-NAME treatment. Data are based on 10 measurement in each group. $* P<0.001 \mathrm{com}-$ pared with crush injury without treatment.

the normal group $(P<0.05)$. No changes were detected in the CBF.

MAP did not change after muscle crush injury (data not shown). Treatment with L-NAME significantly increased MAP $(P<0.001)$.

Determination of NOS isoforms. Fig. 6 demonstrates the expression of the three NOS isoforms in normal and crushed untreated limbs. In normal limb expression of nNOS and eNOS, mRNAs were detected in all samples, whereas iNOS mRNA was on the verge of detection. However, a striking induction of iNOS was observed in the crushed limb, which was maximal at $6 \mathrm{~h}$ after the muscle crush. nNOS expression in the crushed limb did not change from normal, and eNOS slightly increased at 24,48 , and $72 \mathrm{~h}$ after crush.

Fig. 7 shows the density ratio of nNOS, eNOS, and iNOS, normalized to G3PDH. The expression of iNOS in the crushed limb was significantly higher $(P<0.01)$ compared with the

normal limb, at all time points that were measured. $6 \mathrm{~h}$ after the crush, the density ratio of iNOS in the crushed limb was $305 \%$ higher than that of the normal limb (Fig. $7 C$ ). In parallel, eNOS mRNA levels increased significantly $(P<0.05)$ in the crushed limb at 24,48 , and $72 \mathrm{~h}$ after the induced crush (Fig. $7 \mathrm{~B}$ ). In contrast, the expression of nNOS was comparable in both limbs and did not change significantly after crush (Fig. $7 A$ ).

Fig. 8 demonstrates the immunoreactive levels of the various NOS isoforms. Immunoreactive levels of nNOS and eNOS proteins were detected in normal limb. iNOS was undetectable. In line with the alterations in mRNA levels, Western blot analysis revealed parallel changes in the immunoreactive levels of all three NOS isoforms in the crushed limb.

\section{Discussion}

The findings of this study provide new insight into the cellular mechanism of muscle damage in rats after sustained mechanical pressure of skeletal muscle. For the first time we demonstrated that $(a)$ the NO system is involved in the mechanism of muscle crush injury; $(b)$ this involvement is primarily related to a selective induction of iNOS mRNA and increased immunoreactive iNOS levels in the injured muscle; and $(c)$ the increased NO contributes to the hemodynamic changes in the injured muscle after crush. The latter is manifested by extensive vasodilatation leading to muscle hyperperfusion.

The increased NO in the crushed limb that was found in our study might have a physiological role since NO modulates vascular diameter and therefore causes vasodilatation and increased perfusion, which is essential for remodeling the damaged muscle. Moreover, NO may decrease capillary membrane permeability (20). However, the excessive amount of $\mathrm{NO}$ in the tissue has destructive properties which may augment the crush myopathy. Such muscle injury could further increase NO formation, thus creating a vicious cycle and positive feedback.

A major known function of $\mathrm{NO}$ is the regulation of blood

\section{bNOS}

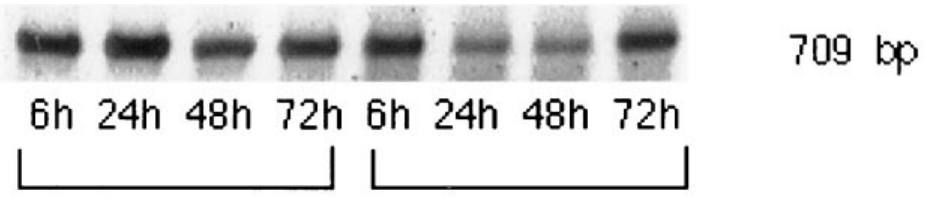

eNDS

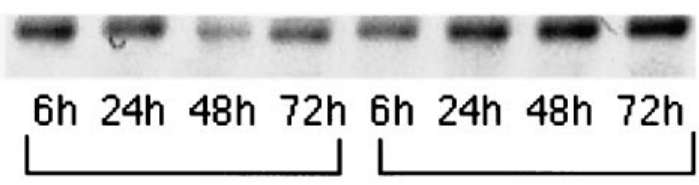

iNOS

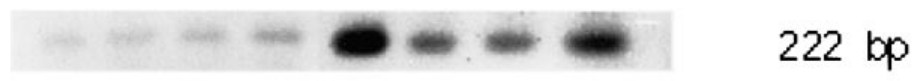

6h $24 h 48$ h $72 h$ 6h $24 h 48 h 72 h$

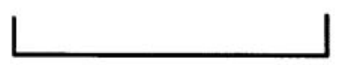

normal limb crushed limb

\section{4 bp}

Figure 6. Representative gel photograph of PCR-amplified nNOS, eNOS, and iNOS mRNA, in intact and crushed tibialis anterior muscle $6,24,48$, and $72 \mathrm{~h}$ after experimental muscle crush. The RT-PCR products were separated on $1 \%$ agarose gel electrophoresis and visualized by ethidium bromide. The sizes of the base pairs are indicated on the right. $b N O S=$ nNOS. 


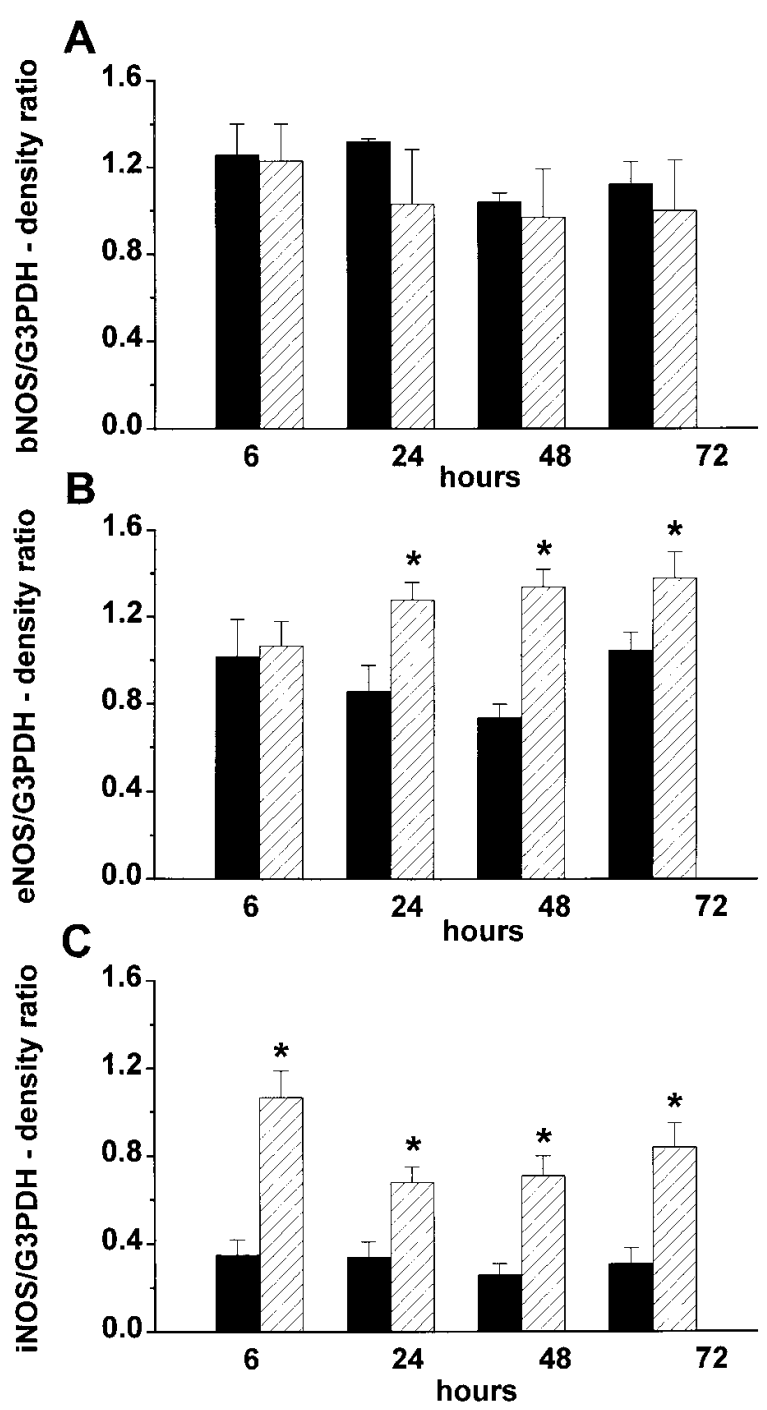

Figure 7. Bar graph showing relative amount of nNOS $(A ; n=5)$, eNOS $(B ; n=8)$, and iNOS $(C ; n=8)$ mRNA, quantified by densitometry and expressed as mean NOS isoform mRNA:G3PDH mRNA ratio. Error bars represent SEM. The black bars indicate intact limb, and the white bars indicate crushed limb. Each pair of bars represents the ratio at different time points: $6,24,48$, and $72 \mathrm{~h}$ after muscle crush. $* P<0.05 ; * * P<0.01$ compared with intact limb at the same time point.

vessel tone and therefore blood flow $(8,9)$. We have shown that crush caused a threefold increase in the capillary mean cross-sectional area, and a three- to fourfold increase in the FBF and CBF. CBF was higher because it was restricted to the crushed muscle itself, whereas FBF estimated global blood flow to the entire limb (crushed and noncrushed areas).

Another function of NO is to regulate the contractile force of skeletal muscles $(6,7)$. Previously, we showed a steep reduction in the contractile force of tibialis anterior muscle after crush injury in the rat (21). Contractility was estimated by the maximal amplitude of the muscular twitch obtained in response to electrical pulse. In the crushed limb, direct stimulation to the fibers showed a decrease of $52 \%$ in mean contractile force compared with the contralateral intact limb. These data are compatible with the known property of NO to regulate contractile force in skeletal muscle $(6,7)$, therefore supporting our speculation of the involvement of the NO system in the pathogenesis of crush.

The mechanism of NO action in skeletal muscle is poorly understood. It is known from the literature that myocyte function is sensitive to redox modulation under basal conditions. Reactive oxygen intermediates (RIO) and NO are two classes of molecules that modulate muscle function under physiological conditions (22). The RIO promote excitation-contraction coupling and appear to be obligatory to optimal contractile function $(22,23)$. NO opposes the action of RIO. The dynamic balance between production and inactivation of RIO and NO maintains redox homeostasis within the cell. NOS activity has a significant influence on the effectiveness of excitation-contraction coupling. Inhibitors of NOS increase twitch and submaximal tetanic force production in skeletal muscles, whereas NO donors decrease force production (6). In smooth muscle and in the diaphragm of the rat, NO acts via cGMP to inhibit muscle contraction. However, overall magnitude of cGMPdependent changes appear to be small. The minor changes in cGMP magnitude suggest additional mechanisms like reversible nitrosylation of redox-active sites on regulatory proteins. NO may exert antioxidant effects by inhibiting oxidation of thiol groups and metal centers by RIO.

Our speculation regarding the involvement of the NO system in muscle crush injury is strengthened by the determination of NOS isoforms. Skeletal muscle cell was the first cell type in which constitutive coexpression of two NOS isoforms was demonstrated $(6,7,10)$; nNOS is localized on or near cell membrane in fast fibers, and eNOS is colocalized with mitochondria. Both isoforms are known to regulate contractility and vascular tone under physiological conditions and were detected in this study as well. However, the most important finding in our study was the finding of the prominent expression of the inducible form of the enzyme. In the normal rat skeletal muscle, iNOS was almost undetectable and was overexpressed only after crush. This suggests the causal involvement of the NO system in the process of muscle crush injury.

iNOS is induced by macrophages, by certain cytokines like TNF and interleukins, and by LPS, which acts through the release of cytokines. Cytokines regulate iNOS, some promoting and others inhibiting the induction of the enzyme (24). We have demonstrated that the crushed muscle contains macrophages and therefore has the potential to induce iNOS. The NO that is produced by iNOS may exert an autocytotoxic effect, as well as a cytotoxic effect on other cells in the vicinity (8, 24). The induction of iNOS in the macrophages, which is inhibited by glucocorticoids, results in the sustained production of NO. There, the NO combines with iron-sulfur centers in key enzymes of both the respiratory cycle and the DNA synthesis pathway. In normal situations, NO is capable of rapidly and reversibly inhibiting the mitochondrial respiratory chain. However, when NO is generated by iNOS, it may change the pattern of the inhibition to a prolonged one $(25,26)$. This may cause cellular hypoxia, in spite of intact circulation.

Considering the properties of overexpression of NO, the remarkable increase in the levels of iNOS mRNA as soon as $6 \mathrm{~h}$ after crush (305\% higher than normal) may explain the triggering of the myopathy in our model and perhaps in crush syndrome in humans. The iNOS protein appears only several hours later because it is undetectable in normal muscle and it 
bNOS

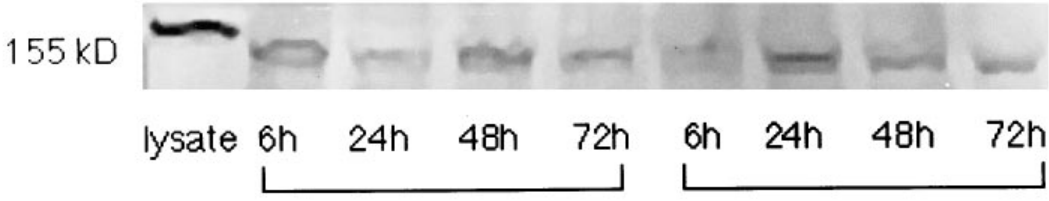

eNOS

$140 \mathrm{kD}$

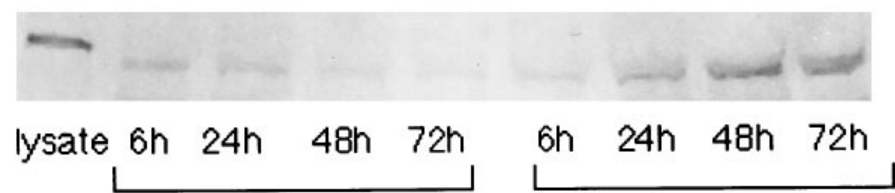

iNOS

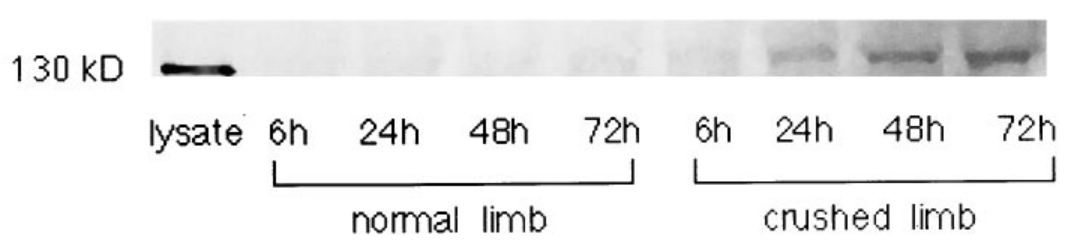

Figure 8. Western blot showing nNOS, eNOS, and iNOS in intact and crushed tibialis anterior muscle $6,24,48$, and $72 \mathrm{~h}$ after muscle crush. Rat brain lysate indicates enriched nNOS, human endothelial lysate indicates enriched eNOS, and mouse macrophage lysate indicates enriched iNOS. The sizes of the molecules in kilodaltons are indicated on the left. $b N O S=\mathrm{nNOS}$. takes a few hours to translate it from mRNA. In addition to iNOS, we found an increase in mRNA and protein levels of eNOS. The increased eNOS will contribute more NO to the system, but is negligible compared with the excessive quantity of NO produced by iNOS. The reason for the increased eNOS may be related to the process of restoration in the damaged tissue, which produces connective tissue and endothelial cells as well.

Excessive generation of NO by the iNOS leads to vasodilatation that is resistant to vasoconstrictors (8). Furthermore, in endotoxin shock in animals the increase in generation of $\mathrm{NO}$ is directly related to the degree of hypotension. Inhibitors of NOS can reverse or prevent the hypotension induced in animals by LPS or TNF and the hypotension in animals with hemorrhagic and anaphylactic shock (8).

Similar hemodynamic characteristics were found in the crushed limb of the rat model: excessive NO formation, remarkable capillary vasodilatation, and muscle hyperperfusion. Treatment with L-NAME prevented the vasodilatation and ameliorated the hyperperfusion associated with crush. The fact that there was no significant change in $\mathrm{CBF}$ after treatment with D-NAME is verifying the specificity of L-NAME as NOS inhibitor in muscle crush injury. L-NAME had a systemic effect due to inhibition of NOS isoforms, leading to decreased NO formation. Such NO suppression in our model reduced the FBF in the contralateral uninjured limb. Furthermore, L-NAME caused an increase in MAP, which theoretically should increase muscle blood flow. However, local impact of the inhibition of NOS apparently overrode the increased MAP and diminished the flow in the normal muscle.

Our hypothesis on the mechanism of crush injury is that the damage occurs due to the external mechanical pressure, which activates nonselective cation channels and causes severe electrolyte disorders. Traditionally, the damage has been attributed to ischemia followed by hypoxia caused by decreased perfusion to the crushed limb. From the data reported in our study, it seems that muscle crush injury is not related either to capillary occlusion or to decrease in muscle perfusion. Crush injury is characterized by extreme vasodilatation in the injured muscle, which causes increased perfusion to the whole limb. The hypothesis of pressure-induced damage in skeletal muscle is not a new one. Heppenstall et al. (27) showed the tolerance of dog skeletal muscle to total warm ischemia applied by a tourniquet for $3 \mathrm{~h}$ and, in contrast, its susceptibility to mechanical pressure for less than $1 \mathrm{~h}$. They found that the metabolic and ultrastructural cellular degeneration was more severe in the group with compartment syndrome in which the intramuscular pressure is high. The levels of muscular ATP diminished rapidly in the animals with compartment syndrome, but remained unchanged in the tourniquet group.

Our results lead to the conclusion that, at least in the rat, the NO system is highly involved in muscle crush injury. NO induction is an important factor in the mechanism of the damage. The selective generation of iNOS and augmentation of eNOS may lead to hypotension and may also cause cellular hypoxia, due to inhibition of the mitochondrial respiratory chain $(25,26)$. Furthermore, NO-dependent arterial hypotension could aggravate the hypovolemic shock that is characteristic of full-blown crush syndrome in humans.

Our findings in the rat clearly suggest that the vasodilatation in the injured skeletal muscle is induced by excessive NO production. It is conceivable that similar events may occur in muscles of humans suffering from crush syndrome. Indeed casualties rescued after entrapment under fallen debris have bleeding tendency in crushed muscles (4). However, extrapolation from rat to man should be taken with caution for the following reasons: first, species differences might exist in the transcriptional regulation of iNOS (28). Second, the rat has slender limbs with thin muscles as compared with the relatively muscular human, whose total muscle mass may approach (or in some athletes exceeds) $50 \%$ of body weight. Thus, our rat model focuses on the events in the skeletal muscle rather than on the systemic consequences of crush syndrome such as hypovolemic shock and myoglobinuric acute renal failure. It is possible, however, that with widespread muscle crush injury in humans, extensive vasodilatation occurs etiologically similar to 
that seen in the rat. Such vasodilatation would aggravate the hypovolemic shock to which these patients are prone. Moreover, induction of NOS by cytokines may not be restricted only to the injured muscle, but may occur in other organs as well, e.g., the kidney, which is commonly involved in crush syndrome in humans. Thus, excessive production of NO via iNOS in the kidney has tubular cytotoxic effects and aggravates renal failure in rats subjected to renal ischemia (29). Furthermore, myoglobin released from crushed muscles in the early stages of injury is nephrotoxic by itself, particularly during aciduria, and by scavenging of NO released from endothelium may aggravate the renal vasoconstriction on the background of hypovolemic shock (30). Therefore, it appears that disturbances in NO regulation in trauma have profound effect on systemic and renal perfusion.

Our study suggests that NOS inhibitors, especially specific iNOS inhibitors or NO scavengers, may have therapeutic potential in patients suffering from crush injury.

\section{Acknowledgments}

The authors are grateful to Mrs. Eva Shuranyi for her expert technical assistance.

This study was supported by grant 2805 from the Israel Ministry of Health.

\section{References}

1. Owen, C.A. 1981. The crush syndrome. In Compartment Syndromes and Volkmann's Contracture. S.J. Mubarak and A.R. Hargens, editors. W.B. Saunders Company, Philadelphia. 166-182.

2. Bywaters, E.G.L. 1990. Fifty years on: the crush syndrome. Br. Med. J. 301:1412-1415.

3. Better, O.S., I. Rubinstein, and J. Winaver. 1992. Recent insights into the pathogenesis and early management of the crush syndrome. Sem. Nephrol. 12: 217-222.

4. Better, O.S., and J.H. Stein. 1990. Early management of shock and prophylaxis of acute renal failure in traumatic rhabdomyolysis. N. Engl. J. Med. 322:825-829.

5. Better, O.S., Z. Abassi, I. Rubinstein, S. Marom, J. Winaver, and M. Silbermann. 1990. The mechanism of muscle injury in the crush syndrome: ischemic versus pressure-stretch myopathy. Miner. Electrolyte Metab. 16:181-184.

6. Kobzik, L., M.B. Reid, D.S. Bredt, and J.S. Stamler. 1994. Nitric oxide in skeletal muscle. Nature. 372:546-548.

7. Kobzik, L., B. Stringer, J.-L. Balligand, M.B. Reid, and J.S. Stamler. 1995. Endothelial type nitric oxide synthase in skeletal muscle fibers: mitochondrial relationships. Biochem. Biophys. Res. Commun. 211:375-381.

8. Moncada, S., and A. Higgs. 1993. The L-arginine-nitric oxide pathway. $N$. Engl. J. Med. 329:2002-2012.

9. Moncada, S., and A. Higgs. 1991. Endogenous nitric oxide: physiology, pathology and clinical relevance. Eur. J. Clin. Invest. 21:361-374.

10. Frandsen, U., M. Lopez-Figueroa, and Y. Hellsten. 1996. Localization of nitric oxide synthase in human skeletal muscle. Biochem. Biophys. Res. Com- mun. 227:88-93

11. Bates, T.E., A. Loesch, G. Burnstock, and J.B. Clark. 1996. Mitochondrial nitric oxide synthase: a ubiquitous regulator of oxidative phosphorylation? Biochem. Biophys. Res. Commun. 218:40-44.

12. Thompson, M., L. Becker, D. Bryant, G. Williams, D. Levin, L. Margraf, and B.P. Goroir. 1996. Expression of the inducible nitric oxide synthase gene in diaphragm and skeletal muscle. J. Appl. Physiol. 81:2415-2420.

13. Brenman, J.E., D.S. Chao, H. Xia, K. Aldape, and D.S. Bredt. 1995. Nitric oxide synthase complexed Dystrophin and absent from skeletal muscle sarcolemma in Duchenne muscular dystrophy. Cell. 82:743-752.

14. Anggard, E. 1994. Nitric oxide: mediator, murderer and medicine. Lancet (N. Am. Ed.). 343:1199-1206.

15. Peterson, D.A., D.C. Peterson, S. Archer, and K.E. Weir. 1992. The non specificity of specific nitric oxide synthase inhibitors. Biochem. Biophys. Res. Commun. 187:797-801.

16. Plyley, M.J., and A.C. Groom. 1975. Geometrical distribution of capillaries in mammalian strained muscle. Am. J. Physiol. 228:1376.

17. Chomczyski, P., and N. Sacchi. 1987. Single-step method of RNA isolation by acid guanidinium thiocyanate-phenol-chloroform extraction. Anal. Biochem. 162:156-159.

18. Tso, Y., X.-H. Sun, T.-H. Kao, K.S. Rees, and R. Wu. 1985. Isolation and characterization of rat and human glyceraldehyde-3-phosphate dehydrogenase cDNAs: genomic complexity and molecular evolution of the gene. Nucleic Acids Res. 13:2485-2502.

19. Har-Shai, Y., M. Silbermann, N.D. Reis, C. Zinman, I. Rubinstein, Z. Abassi, and O.S. Better. 1992. Muscle microcirculatory impairment following acute compartment syndrome in the dog. Plas. Reconstr. Surg. 89:283-289.

20. Kubes, P. 1993. Nitric oxide-induced microvascular permeability alterations: a regulatory role for cGMP. Am. J. Physiol. 265:H1909-H1915.

21. Rubinstein, I., D. Kerem, Y. Melamed, and O.S. Better. 1993. Hyperbaric $\mathrm{O} 2$ (HBO) and mannitol (M) enhance recovery from muscular crush injury. In Proceedings of the XIXth Annual Meeting of EUBS. R.E. Reinertsen, A.O. Brubakk, and G. Bolstad, editors. SINTEF UNIMED, N-7034. Trondheim, Norway. 137-141.

22. Reid, M.B. 1996. Reactive oxygen and nitric oxide in skeletal muscle. News Physiol. Sci. 11:114-119.

23. Reid, M.B., F.A. Khawli, and M.R. Moody. 1993. Reactive oxygen in skeletal muscle. III. Contractility of unfatigued muscle. J. Appl. Physiol. 75: 1081-1087.

24. Moncada, S., and A. Higgs. 1995. Molecular mechanisms and therapeutic strategies related to nitric oxide. FASEB (Fed. Am. Soc. Exp. Biol.) J. 9:1319-1330.

25. Cleeter, M.W.J., J.M. Cooper, V.M. Drley-Usmar, S. Moncada, and A.H.V. Schapira. 1994. Reversible inhibition of cytochrome C oxidase, the terminal enzyme of the mitochondrial respiratory chain, by nitric oxide. Implications for neurodegenerative diseases. FEBS (Fed. Eur. Biochem. Soc.) Lett. 345:50-54.

26. Brown, G.C., and C.E. Cooper. 1994. Nanomolar concentrations of nitric oxide reversibly inhibit synaptosomal respiration by competing with oxygen at cytochrome oxidase. FEBS (Fed. Eur. Biochem. Soc.) Lett. 345:50-54.

27. Heppenstall, R.B., R. Scott, A. Sapega, Y.S. Park, and B. Chance. 1986. A comparative study of the tolerance of skeletal muscle to ischemia. J. Bone Jt. Surg. Am. Vol. 68-A:820-828.

28. de Vera, M.E., R.A. Shapiro, A.K. Nussler, J.S. Mudgett, R.L. Simmons, S.M. Morris, Jr., T.R. Billiar, and D.A. Geller. 1996. Transcriptional regulation of human inducible nitric oxide synthase (NOS2) gene by cytokines: initial analysis of human NOS2 promotor. Proc. Natl. Acad. Sci. USA. 93:1054 1059.

29. Noiri, E., T. Peresleni, F. Miller, and M. Goligorsky. 1996. In vivo targeting of inducible NO synthase with oligodeoxynucleotides protects rat kidney against ischemia. J. Clin. Invest. 97:2377-2383.

30. Zager, R.A. 1996. Rhabdomyolysis and myohemoglobinuric acute renal failure. Kidney Int. 49:314-316. 